\title{
Effect of probiotics on five biochemical microflora-associated characteristics, in vitro and in vivo
}

\author{
Maria E. Cardona', Vibeke de V. Vanay², Tore Midtvedt² and Elisabeth \\ Norin' \\ 'Microbiology and Tumor Biology Center. \\ ${ }^{2}$ Department of Cell and Molecular Biology, Laboratory of Medical Microbial Ecology, Stockholm, Sweden.
}

Abstract

Background: Several bacterial strains, mainly those belonging to the genera Lactobacillus, Enterococcus, Streptococcus and Bifidobacterium, are currently used as probiotics.

Objective: The aim of this study was to investigate the influence of probiotics on five biochemical microbial-related functions in vitro and in vivo, in gnotobiotic mice, by applying the GAC/MAC (germ-free animal characteristic/microflora-associated characteristic) concept.

Design: Sixteen probiotics were monoinoculated in vitro and/or monoassociated to germ-free mice to investigate the following biochemical parameters in large intestinal samples: inactivation of tryptic activity, degradation of $\beta$-aspartylglycine and of mucin, conversion of bilirubin to urobilinogen and $\beta$-glucuronidase activity.

Results: Lactobacillus reuteri 2010, L. rhamnosus strain 271, L. rhamnosus ATCC7469 and L. rhamnosus GG ATCC53103 expressed $\beta$-glucuronidase activity. The other parameters tested were not influenced by any of the probiotics.

Conclusions: Bacterial $\beta$-glucuronidases have an important intestinal function. As many microbial-host and microbe-diet interactions occur in the gastrointestinal tract of mammals, microbial-related functions should be studied in greater detail in probiotics.

Keywords: Germ-free, germ-free animal characteristic, $\beta$-glucuronidases, gnotobiotic mice, microflora-associated characteristic, probiotics.

Received: October 16, 2001. Revisions: January 5, 2002. February 20, 2002. Accepted: April $23,2002$.

\section{Introduction}

Several bacterial strains, mainly those belonging to the genera Lactobacillus, Enterococcus, Streptococcus and Bifidobacterium, are currently used as probiotics, defined as "live microbial feed supplements which beneficially affect the host, by improving its intestinal microbial balance" (1).

Over the years, many health claims have been related to intake of probiotics (for review see Refs 2-4). However, the mechanisms of action in the gastrointestinal tract are not well known and relatively little is known about their biochemical performance. Comparisons of conventionally raised (CV) mammals with their germ-free (GF) counterparts have revealed a series of anatomical, biochemical, immunological and physiological phenotypes collectively known as microflora-asso- ciated characteristics (MACs). In the absence of the functionally active micro-organisms, the phenotype observed is defined as germ-free animal characteristic (GAC) (5). The microflora exerts an influence on faecal tryptic activity (FTA), $\beta$-aspartylglycine, intestinal mucin and bilirubin metabolism, among other biochemical parameters.

FTA represents the net sum of several interactions involving the secretion of trypsinogen and trypsin inactivators from the pancreas, activation of trypsinogen to trypsin, and the intestinal presence of microbial- and diet-derived compounds that may inactivate trypsin. GF rodents always excrete high levels of FTA, whereas $\mathrm{CV}$ rodents excrete little or no FTA (6). Inactivation of FTA was followed in this investigation to study pancreatic/intestinal flora interactions. 
$\beta$-Aspartylglycine is a member of a group of $\beta$-carboxyl dipeptides in the intestinal tract, formed from dietary proteins by host-derived proteolytic enzymes. The $\beta$-carboxyl bonds are assumed to be cleaved only by proteases derived from microbes. $\beta$-Aspartylglycine was initially detected in caecal contents from GF mice, whereas it was not present in the $\mathrm{CV}$ controls. Further studies showed that association of GF mice with an increasing number of anaerobic intestinal bacterial strains leads to a gradual reduction of $\beta$-aspartylglycine in faeces. $\beta$-Aspartylglycine has been suggested as an indicator of "colonization resistance" (7). Therefore, the presence of $\beta$-aspartylglycine in the faeces of humans or $\mathrm{CV}$ mammals indicates that the normal intestinal microbial ecosystem is markedly altered.

The gastrointestinal mucin, produced by goblet cells and glandular mucous cells, comprises a polypeptide core with oligosaccharide side-chains linked by $o$-glycosidic bonds. Mucin plays many physiological and pathophysiological roles in the intestine of mammals (8). Mucin is degraded in $\mathrm{CV}$ animals, while it is undegraded in GF animals. Carlstedt-Duke et al. (9) isolated a Peptostreptococcus strain able to obliterate all gel electrophoretic mucin bands completely, both in vitro and in vivo. Degradation of mucin was followed to study an intestinal function of major importance in preserving the integrity of the intestinal mucosa.

Bilirubin is derived from the catabolism of haemoglobin and some haem-containing compounds. In the liver, bilirubin is conjugated to glucuronate and the conjugates are secreted into the bile. Following secretion into the intestine, the bilirubin glucuronides undergo deconjugation and transformation by microbial enzymes to a series of urobilinogens and related products, collectively termed urobilinogen. GF animals do not transform bilirubin to urobilinogen, whereas $\mathrm{CV}$ animals do (10). Until recently only one bacterium had been implicated in that transformation $(10,11)$.

The deconjugation of bilirubin by microbial $\beta$ glucuronidases represents another MAC. Many xenobiotics are eliminated from the body by conjugation with glucuronic acid, forming glucuronides. Deconjugation of glucuronides in the intestine is performed by $\beta$-glucuronidases. Some intestinal $\beta$ glucuronidases may be derived from endogenous sources, but most are of microbial origin (12). GF animals show much lower levels of $\beta$-glucuronidase activity in their faeces than do $\mathrm{CV}$ animals.
Conversion of bilirubin to urobilinogen and $\beta$ glucuronidase activity were followed to investigate hepatic/intestinal flora interactions.

The aim of this study was to investigate the influence of some probiotic strains on the MACs described above, in vitro and in vivo, in monoassociated mice. Sixteen probiotic strains, most of them used commercially in Europe, were analysed.

\section{Material and methods}

\section{Bacterial strains}

The strains investigated in this study are listed in Table 1. Most of them were either purchased from international collections or kindly provided as free gifts from various sources (13). Two of the strains, Clostridium ramosum G62 and Escherichia coli X7, were used as positive controls for conversion of bilirubin to urobilinogen and $\beta$-glucuronidase activity, respectively.

Before the in vitro and the in vivo inoculations, aliquots of each bacterium were cultured in their respective growth media (Table 1) and incubated anaerobically at $37^{\circ} \mathrm{C}$ for $72 \mathrm{~h}$. Then, for the in vivo inoculations, aliquots of $10 \mathrm{ml}$ were dispensed into ampoules, which were sealed and sterilized on the outside with chromsulfuric acid, before being transferred to the GF isolator.

\section{A nimals}

Eighty GF and nine CV mice, of the strain NMRI$\mathrm{KI}$, around 75 days old of both sexes, were used. From the total GF animals, 71 were allotted to 18 groups comprising four or five mice each. Sixteen groups were monoassociated with the respective probiotic and one group with C. ramosum G62. The remaining nine GF mice and the $\mathrm{CV}$ animals were used as controls.

The GF mice were reared in lightweight stainless-steel isolators and the CV mice in an ordinary animal room with artificial light between 06:00 and 18:00, temperature $24 \pm 2^{\circ} \mathrm{C}$ and humidity $55 \pm$ $10 \%$. All animals were fed an autoclaved rodent diet (R36 Lactamin, Sweden) and had free access to water. The study was approved by the local ethics committee for animal research, Sweden.

\section{Monoassociation procedure}

Each mouse group was transferred into a small stainless-steel rearing isolator (SRI) together with the ampoule containing the bacterium. The ampoule was broken inside the SRI and the bacterial 
Table I. Bacterial strains tested in vitro and/or in vivo, as monoassociates in gnotobiotic mice, and their corresponding $\beta$-glucuronidase activity in vitro

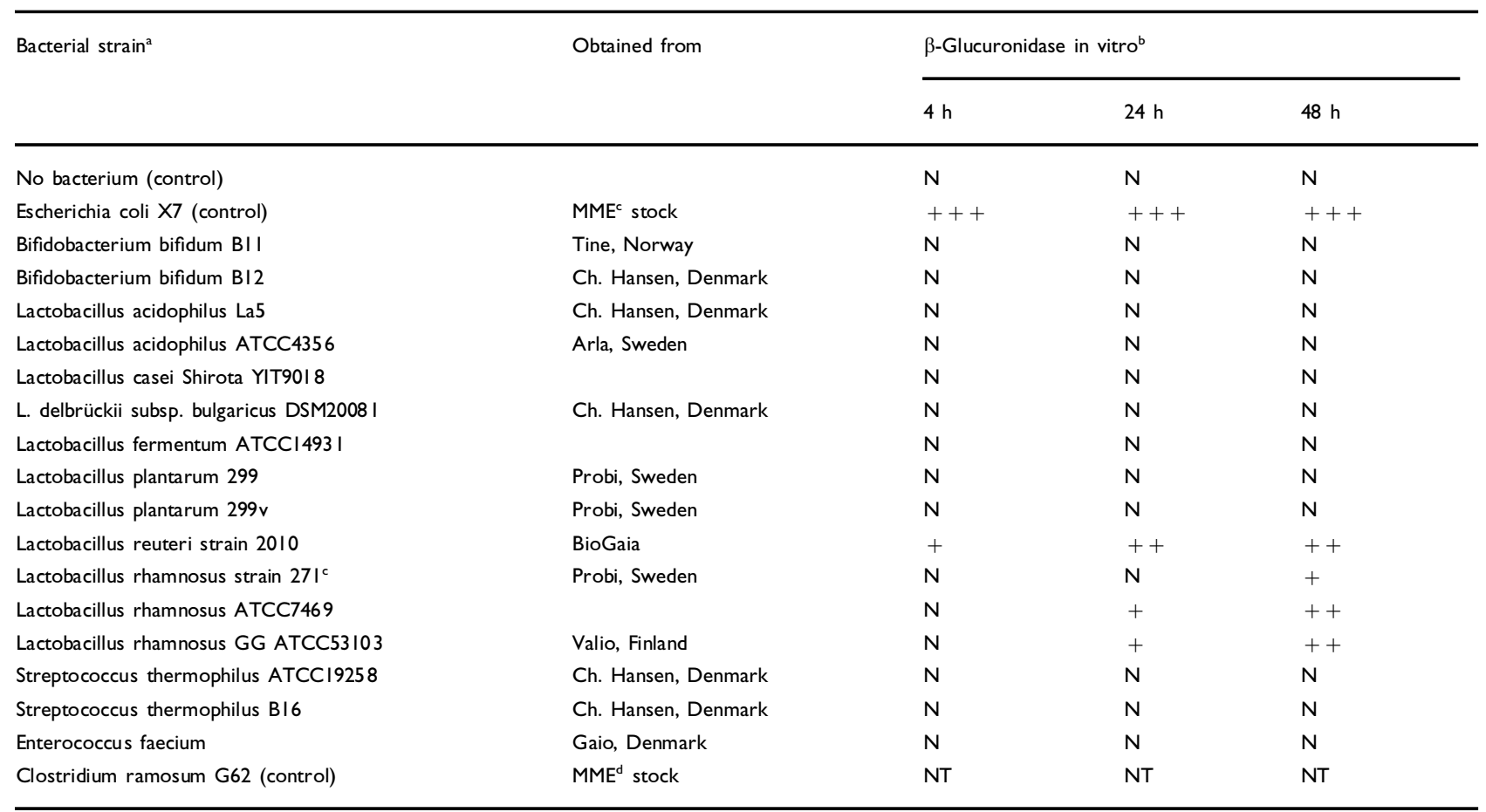

${ }^{a}$ E coli X7, S. thermophilus ATCC19258 and C. ramosum G62 were grown in Glucose (Difco, USA), Todd Hewitt (Difco, USA) broth and in Reinforced Clostridial medium (Merck, Germany), respectively. The other strains were grown in MRS broth (Merck, Germany).

${ }^{\mathrm{b}} \beta$-Glucuronidase activity was assayed qualitatively by the $\mathrm{p}$-nitrophenyl method (PGUA tablets). Results after 4, 24 and $48 \mathrm{~h}$ incubation are given as $\mathrm{N}$ : no enzyme activity (colourless); + : weak positive reaction; ++ : clear positive reaction; +++ : strong positive reaction (strong yellow colour); NT: not tested.

' The strain was labelled as L. plantarum strain 27 I on receipt.

${ }^{d}$ Laboratory of Medical Microbial Ecology.

suspension was spread on the bedding material and fur of the mice. The animals remained in the SRI for 10-15 days. Then, they were taken out and killed by cervical dislocation. Establishment and number of bacteria were ensured by taking samples from the caecum into appropriate broth media and spreading on to agar plates, followed by anaerobic incubation at $37^{\circ} \mathrm{C}$. Growth was inspected for at least $72 \mathrm{~h}$. The total caecum and colon content from each mouse was stored in a closed vial at $-20^{\circ} \mathrm{C}$ for biochemical analyses.

\section{Biochemical analyses}

Except for differences in preparing the aliquots to be tested, the methods were performed similarly in the in vitro and in the in vivo part of this investigation.

\section{In vitro}

Medium. A medium to analyse both FTA and $\beta$-aspartylglycine was prepared. GF AGUS rat faeces plus sterile Todd Hewitt medium (1:5) were thoroughly mixed and filtered. This proce- dure was carried out in a GF isolator. The mixture was then dispensed in $10 \mathrm{ml}$ tubes and brought out of the isolator. Thereafter, these media were inoculated with aliquots of $0.5 \mathrm{ml}$ from each probiotic culture. These preparations, together with two inoculated controls, were incubated at $37^{\circ} \mathrm{C}$ for $24 \mathrm{~h}$. In addition, two tubes containing the prepared medium were stored at $-20^{\circ} \mathrm{C}$ and used as uninoculated and unincubated controls. The samples were assayed as follows.

Faecal tryptic activity. The assay was performed at room temperature $\left(20^{\circ} \mathrm{C}\right)$. An aliquot of $0.1 \mathrm{ml}$ from the inoculated medium (above) was added to $2.9 \mathrm{ml}$ of Tris buffer ( $\mathrm{pH} 8.2$ ) containing $4.4 \mathrm{~g}$ of calcium chloride per litre. The reaction was initiated by adding $0.6 \mathrm{ml}$ of $0.003 \mathrm{M}$ $N$-benzoyl-DL-arginine- $p$-nitroanilide hydrochloride (BAPNA) and terminated after $10 \mathrm{~min}$ by adding $0.6 \mathrm{ml}$ of $5 \mathrm{M}$ acetic acid. Bovine pancreas trypsin type III diluted in $2 \mathrm{mM}$ hydrochloric acid was used for the construction of a 
standard curve. All samples and standards were analysed in parallel with blanks at $405 \mathrm{~nm}$ on a spectrophotometer (Hitachi 150-20, Tokyo, Japan). Results are given as mg FTA $\mathrm{kg}^{-1}$ faeces.

Degradation of $\beta$-aspartylglycine. Aliquots of $30 \mu \mathrm{l}$ from the inoculated media (above) and from a commercial standard dilution of $\beta$-aspartylglycine $\left(2 \mathrm{mg} \mathrm{ml}^{-1}\right.$ ) were applied on Whatmann $3 \mathrm{MM}$ chromatography paper. Then, high-voltage paper electrophoresis was performed in the buffer pyridine:acetic acid:water (1:10:89), $\mathrm{pH} 3.5$, giving a current of $150 \mathrm{~mA}$ and $100 \mathrm{~W}$ for $1 \mathrm{~h}$. After electrophoresis, the paper was dried at $80^{\circ} \mathrm{C}$ and sprayed with ninhydrin. Heating afterwards at $150^{\circ} \mathrm{C}$ for $10 \mathrm{~min}$ gives a clear blue spot for $\beta$-aspartylglycine. The intensity of the colour of the samples was compared visually with that of the standard and reported as present or absent.

$\beta$-Glucuronidase activity. The activity of $\beta$-glucuronidase was assayed qualitatively by the $p$-nitrophenyl method, using diagnostic PGUA tablets (Rosco Diagnostica, Denmark). A dense bacterial suspension of each probiotic, approx. McFarland No. 4, was prepared in $0.25 \mathrm{ml}$ of phosphate buffer at $\mathrm{pH} 6.5$ and at $\mathrm{pH}$ 8.0. A similar suspension of $E$. coli $\mathrm{X} 7$ and a solution of phosphate buffer without any bacterium were used. A tablet of substrate, $p$-nitrophenyl- $\beta$-D-glucuronide, was added to each tube, followed by incubation at $37^{\circ} \mathrm{C}$. $\beta-\mathrm{Glu}-$ curonidase activity was recorded by the degree of $p$-nitrophenol coloration from negative (colourless) to +++ (strong yellow).

\section{In vivo}

The total caecum and colon content from the monoassociated mice, above, was thawed and homogenized. An aliquot of $0.7 \mathrm{~g}$ was taken for determination of urobilinogen. A second aliquot of 0.5-1 g was diluted with two volumes of saline, homogenized and placed at $4^{\circ} \mathrm{C}$ for $2 \mathrm{~h}$. Thereafter, the homogenate was centrifuged at $4500 \mathrm{rpm}, 4^{\circ} \mathrm{C}$, for $30 \mathrm{~min}$. The supernatant was separately used for the determination of inactivation of FTA, and degradation of $\beta$-aspartylglycine and of mucin.

Faecal tryptic activity. Aliquots of $0.1 \mathrm{ml}$ from the supernatants were assayed as for the in vitro test. Results are expressed as mg of FTA $\mathrm{kg}^{-1}$ large intestinal content.
Degradation of $\beta$-aspartylglycine. Aliquots of $30 \mu 1$ from the supernatants were assayed as for the in vitro test. The results are given as presence or absence. $\beta$-Aspartylglycine is present in faeces from $\mathrm{GF}$ animals, but absent in CV animals.

Degradation of mucin. Aliquots of $5 \mu \mathrm{l}$ from the supernatants were assayed by agar gel electrophoresis on glass plates, using $1 \%$ agarose in sodium barbiturate buffer, $\mathrm{pH}$ 8.6. The electrophoretic separation was run for $30 \mathrm{~min}$ with a potential gradient of $20 \mathrm{~V} \mathrm{~cm}^{-1}$, giving a current of $160 \mathrm{~mA}$. The plate was immediately transferred to a fixing solution containing ethanol:chloroform:acetic acid (6:3:1). After $30 \mathrm{~min}$, it was dried in a warm-air stream. The staining procedures were Toluidine blue (TB) $0.05 \% \mathrm{w} / \mathrm{v}$ in acetate buffer, $\mathrm{pH}$ 5.0, periodic acid-Shiff (PAS) and Coomassie brilliant blue R (CB) $0.05 \% \mathrm{w} / \mathrm{v}$ in $12 \%$ trichloroacetic acid. Faecal samples extracts from $\mathrm{GF}$ and from $\mathrm{CV}$ rats were run on each gel as controls. The samples were compared with the controls and evaluated as follows. An electrophoretic pattern similar to that found in the $\mathrm{CV}$ control (no bands $=0$ ) is presented as degraded; an electrophoretic pattern similar to that found in GF control (presence of bands $=3$ ) is presented as undegraded; and electrophoretic patterns with some bands are scored 1 and 2 .

Conversion of bilirubin to urobilinogen. Urobilinogen was assayed by applying the Ehrlich's aldehyde reaction with $p$-dimethylaminobenzaldehyde directly to an aqueous homogenate of the large intestinal content, after reduction of urobilin to urobilinogen with ferrous sulfate. The procedure was as follows. An aliquot of $0.7 \mathrm{~g}$ from the large intestinal content was homogenized with $4.2 \mathrm{ml}$ of distilled water. Then, $4.7 \mathrm{ml}$ of $1.3 \mathrm{M}$ ferrous sulfate was added and mixed. Thereafter, $4.7 \mathrm{ml}$ of $2.5 \mathrm{M}$ sodium hydroxide were added and shaken vigorously. The mixture was left in darkness at room temperature. After $2 \mathrm{~h}$, it was filtered and centrifuged at $4500 \mathrm{rpm}, 4^{\circ} \mathrm{C}$, for $20 \mathrm{~min}$. Two aliquots of $1 \mathrm{ml}$ from the supernatant were run as test and blank, and read against distilled water on a spectrophotometer (Hitachi 150-20). Phenolsulfonphthalein was used as an artificial standard for "urobilinogen-aldehyde". The values are converted and given as mmol urobilinogen $\mathrm{kg}^{-1}$ large intestinal content (wet weight). 


\section{Results}

\section{In vitro}

As is evident from Table 2, all of the incubations of probiotic strains for inactivation of FTA and degradation of $\beta$-aspartylglycine gave similar results as uninoculated but incubated controls.

Lactobacillus reuteri 2010 , L. rhamnosus strain 271, L. rhamnosus ATCC7469 and L. rhamnosus GG ATCC53103 expressed $\beta$-glucuronidase activity (Table 1). They showed either a weak positive or a clear positive reaction as the incubation increased. A yellowish colour was observed in the initial suspension of $L$. reuteri 2010. However, the colour also became stronger as the incubation increased. To confirm its enzyme activity, $L$. reuteri 2010 was retested using the same amount of substrate ( $p$-nitrophenyl- $\beta$-D-glucuronide, Sigma) contained in the diagnostic tablet previously used. The absorbance of the sample was read at $400 \mathrm{~nm}$ on a spectrophotometer (Hitachi 150-20) and compared with that of $E$. coli X7 strain. A slight positive reaction was observed. No differences were found with respect to $\mathrm{pH} 6.5$ and $\mathrm{pH} 8.0$ (data not given).

\section{In vivo}

All animals remained healthy throughout the study. All of the bacterial strains were established as monoassociates in the intestine of the mice, and all of them except for $L$. delbrückii subsp. bulgari-

Table 2. Biochemical microflora-associated characteristics (MACs) in vitro and in vivo, in germ-free (GF) and conventional (CV) mice and in ex-GF mice monoassociated with a probiotic

\begin{tabular}{|c|c|c|c|c|}
\hline \multirow[t]{2}{*}{ Group $^{a}$} & \multicolumn{2}{|l|}{$\mathrm{FTA}^{\mathrm{b}}$} & \multicolumn{2}{|c|}{$\beta$-Aspartylglycine ${ }^{c}$} \\
\hline & In vitro & In vivo & In vitro & In vivo \\
\hline Control $^{\mathrm{d}}$ & 45 & - & Present & - \\
\hline Probiotic $^{\mathrm{e}}(4-5)$ & $48(40-65)$ & $1007(695-1297)$ & Present & Present \\
\hline GF mice (9) & - & $1091(696-1398)$ & - & Present \\
\hline $\mathrm{CV}$ mice (9) & - & $18(0-27)$ & - & Absent \\
\hline
\end{tabular}

${ }^{a}$ Figures in parentheses are number of animals used.

${ }^{b}$ Figures are medians (maximum and minimum) of milligrams of faecal tryptic activity (FTA) either per litre of medium (in vitro) or per kilogram of large intestinal content (in vivo).

c An electrophoretic pattern showing bands similar to that of the positive control and one showing no bands are given as present and as absent, respectively.

${ }^{d}$ GF AGUS rat faeces plus sterile Todd Hewitt medium.

e The probiotic strains listed in Table I, except for L. casei and L. reuteri, were investigated. cus were counted in numbers higher than $10^{7}$; the number of $L$. delbrückii subsp. bulgaricus was $10^{3}$. In none of the monoassociated animals was contamination observed.

The median value of FTA in all groups of mice monoassociated with a probiotic was similar to that of the GF mice (Table 2). $\beta$-Aspartylglycine was present in all samples from monoassociated mice (Table 2).

In all monoassociated animals the mucin patterns were qualitatively and quantitatively similar to the patterns found in the GF control.

There was no transformation of bilirubin to urobilinogen in any of the mice monoassociated with a probiotic.

\section{Discussion}

In the present study, none of the probiotic bacterial strains was able to inactivate FTA or to degrade $\beta$-aspartylglycine in vitro or in vivo. As stated before, these capabilities seem to be a rare event among intestinal micro-organisms $(6,14,15)$.

All probiotic lactobacilli and bifidobacteria have saccharolytic action. Therefore, it might be reasonable to assume that they may participate in the breakdown of mucin in vivo. However, as stated in the Results section, the band patterns in monoassociated and GF mice were similar. In the present study, three different staining methods were used for the visualization of bands. TB is commonly used for the expression of glycoproteins, PAS is a staining method for neutral glycoproteins, whereas $\mathrm{CB}$ is a staining method for proteins. The methods do not allow the conclusion to be drawn that the probiotic strains have not removed certain sugar residues from major molecules. However, the parallelism in the stainings in GF and monoassociated animals indicates that a great part of the mucin is electrophoretically intact.

None of the probiotics showed an ability to convert bilirubin to urobilinogen in vivo. The microbial species that are performing this reaction in vivo, to the authors' knowledge, are virtually unknown.

Bilirubin as well as some endogenous and many foreign compounds (xenobiotics) are conjugated with glucuronic acid in the liver, to facilitate their elimination. The glucuronides formed are then secreted into the intestine and may undergo hydrolysis. In general, the free compounds are more easily absorbed from the intestinal tract than the conjugated ones. As stressed by Kroemer and Klotz (16), glucuronidation can be viewed as a 
first-line detoxification mechanism. The importance of knowing the presence of $\beta$-glucuronidases in probiotics, can be exemplified as follows. Very recently, several conjugated and unconjugated IX $\alpha$ and IX $\beta$ isomers of bilirubin were found to be present, in various levels, in meconium and the stools from newborns (17). Depending on the type of isomers and degree of conjugation, they might be excreted either by the mother (before delivery) or stored in the meconium (until delivery). At birth, establishment of an intestinal flora capable of deconjugating and/ or further transforming the bilirubin derivatives may influence the enterohepatic circulation and the faecal excretion of these compounds. Therefore, when giving a $\beta$-glucuronidase-producing probiotic strain to a newborn child, this function has to be evaluated. It is well known that bilirubin and some of its derivatives may cause damage to the newborn child.

In the present investigation, $\beta$-glucuronidase activity was expressed in four out of 16 probiotic strains, when tested in vitro. These results are in agreement with previous investigations showing deconjugating activity in some lactobacilli (18): "With the exception of a $L$. reuteri strain which was weakly positive, all other strains that produced $\beta$-glucuronidase belonged to the $L$. rhamnosus species. Whether $\beta$-glucuronidase production is a common trait in this species or not, remains to be determined". Previous studies on $\beta$-glucuronidase enzyme, comparing its activity in vitro, and in vivo in monoassociated animals, have clearly shown that the activity is expressed in vivo when screened to be present in vitro (19, 20). Therefore, it seems reasonable to assume that the strains used in this study will express in vivo activity as well.

Alteration in levels of intestinal microbial $\beta$ glucuronidases may, in addition to bilirubin, influence the metabolism of other glucuronides, such as several procarcinogens and other xenobiotics (21).

\section{Acknowledgements}

The authors are very grateful to Anna-Karin Persson for her excellent technical assistance. We also wish to thank Ewa Österlund, Sandra Andersson and Johannes Bergstedt for their valuable assistance with the animals. We gratefully acknowledge support from Norske Meierier, TINE and Alpharma A/S, Oslo, Norway. This study was financially supported by grants from the Swedish Medical Research Council (16X-06852) and Karolinska Institutet Funds.

\section{References}

1. Fuller R: Probiotics in man and animals. J Appl Bacteriol 1989;66:365-78.

2. McFarland LV: A review of the evidence of health claims for biotherapeutic agents. Microb Ecol Health Dis 2000; 12:65-72.

3. Marteau PR, de Vrese M, Cellier CJ, Schrezenmeir J. Protection from gastrointestinal diseases with the use of probiotics. Am J Clin Nutr 2001; 73 (2 Suppl): 430-6S.

4. Isolauri E: Probiotics in human disease. Am J Clin Nutr 2001;73:1142-1146S.

5. Midtvedt T. Microbial functional activities. In: Hanson LA, Yolken RH, eds. Probiotics, other nutritional factors, and intestinal microflora. Nestlé Nutrition Workshop, 1997, Vol. 42. Philadelphia: Lippincott-Raven, 1999: 79-96.

6. Norin KE, Gustafsson BE, Midtvedt T: Strain differences in faecal tryptic activity of germ-free and conventional rats. Lab Anim 1986;20:67-9.

7. Welling GW, Helmus G, de Vries-Hospers HG, Tonk RHJ, van der Waaij D, Haralambie E, Linzenmeier G: Rationale for use of $\beta$-aspartylglycine as indicator of colonization resistance. Prog Clin Biol Res 1985; 181: 155-8.

8. Corfield AP, Myerscough N, Longman R et al: Mucins and mucosal protection in the gastrointestinal tract: new prospects for mucins in the pathology of gastrointestinal disease. Gut 2000;47:589-94.

9. Carlstedt-Duke B, Midtvedt T, Nord CE, Gustafsson $\mathrm{BE}$ : Isolation and characterization of a mucin-degrading strain of Peptostreptococcus from rat intestinal tract. APMIS B 1986;94:293-300.

10. Midtvedt T, Gustafsson BE: Microbial conversion of bilirubin to urobilins in vitro and in vivo. Acta Pathol Microbiol Scand B 1981;89:57-60.

11. Gustafsson BE, Swenander-Lanke L. Bilirubin and urobilins in germfree, ex-germfree and conventional rats. $\mathrm{J}$ Exp Med 1960; 112: 975-81.

12. Rød TO, Midtvedt T: Origin of intestinal beta-glucuronidase in germfree, monocontaminated and conventional rats. Acta Pathol Microbiol Scand B 1977;85:27 1-6.

13. Cardona ME, de Vibe V, Midtvedt T, Norin KE: Probiotics in gnotobiotic mice. Conversion of cholesterol to coprostanol in vitro and in vivo and bile acid deconjugation in vitro. Microb Ecol Health Dis 2000;12:219-24.

14. Welling GW, Groen G, Tuinte JH et al: Biochemical effects on germ-free mice of association with several strains of anaerobic bacteria. J Gen Microbiol 1980;117:57-63.

15. Ramare F, Hautefort I, Verhe F et al: Inactivation of tryptic activity by a human-derived strain of $\mathrm{Bac}$ teroides distasonis in the large intestines of gnotobiotic rats and mice. Appl Environ Microbiol 1996;62:1434-6.

16. Kroemer HK, Klotz U. Glucuronidation of drugs. A re-evaluation of the pharmacological significance of the 
conjugates and modulating factors. Clin Pharmacokinet 1992; 23: 292-310.

17. Aziz S, Kotal P, Leroy $P$ et al: Bilirubin-IXalpha and -IXbeta pigments, coproporphyrins and bile acids in meconium and stools from full-term and preterm neonates during the first month of life. Acta Paediatr 2001;90:81-7.

18. Hawksworth G, Drasar BS, Hill MJ: Intestinal bacteria and the hydrolysis of glycosidic bonds. J Med Microbiol 1971;4:451-9.

19. Gadelle D, Raibaud P, Sacquet E: Beta-glucuronidase activities of intestinal bacteria determined both in vitro and in vivo in gnotobiotic rats. Appl Environ Microbiol 1985;49:682-5.
20. Tamura M, Hirayama K, Itoh K: Comparison of colonic bacterial enzymes in gnotobiotic mice monoassociated with different intestinal bacteria. Microb Ecol Health Dis 1996;9:287-94.

21. Hughes R, Rowland IR. Metabolic activities of the gut microflora in relation to cancer. Microb Ecol Health Dis 2000; 2 (Suppl): 179-85.

\section{Maria E. Cardona}

Microbiology and Tumor Biology Center, Laboratory of Medical Microbial Ecology, von Eulers väg 5, Karolinska Institutet, SE-17I 77 Stockholm, Sweden.

E-mail: maria.cardona@mtc.ki.se 